\title{
EL TEATRO COMO RECURSO PARA AFRONTAR LOS RETOS DE LA ADOLESCENCIA
}

\author{
Recepción: 11/04/2018 | Revisión: 11/07/2018 | Aceptación: 28/08/2018
}

Tomás MOTOS TERUEL

Universitat de València

tomas.motos@uv.es

\author{
Vicente ALFONSO-BENLLIURE \\ Universitat de València \\ vicente.alfonso@uv.es
}

Resumen: El presente estudio, utilizando una metodología de investigación cualitativa, intenta profundizar en las creencias implícitas y percepciones que los adolescentes y jóvenes tienen sobre sus experiencias como participantes activos en actividades teatrales. Este artículo se enmarca en un proyecto de investigación (El Teatro y los Jóvenes) estructurado en varias fases y que combina metodologías cuantitativas y cualitativas. Un total de 53 sujetos de edades comprendidas entre los 12 y los 20 años participaron en el estudio. Estos fueron distribuidos al azar en 5 grupos de discusión. Las sesiones de trabajo fueron grabadas, transcritas y posteriormente analizadas, lo que permitió la identificación de categorías, subcategorías y unidades temáticas. Los resultados ponen de manifiesto que las principales opiniones y preocupaciones de los adolescentes respecto al teatro giran en torno a la influencia sobre su propio desarrollo personal, su autoconfianza y competencia emocional (plano individual) y sobre el efecto que las actividades dramáticas tienen sobre sus habilidades personales y empáticas (plano social). Finalmente, se discuten los resultados en clave educativa, tanto a nivel de diseño curricular como de metodología docente.

Palabras clave: teatro; adolescencia; desarrollo positivo; grupos de discusión; metodología cualitativa.

\section{ACTING: A RESOURCETO FACETHE CHALLENGES OFADOLESCENCE}

Abstract: The present study, using qualitative research methodology, tries to deepen in the implicit beliefs and perceptions that young people have about their experiences as active participants in theatrical activities. This paper is part of a research project (Theatre and Young People) structured in several phases that combines quantitative and qualitative methodologies. A total of 53 subjects between the ages of 12 and 20 participated in the study. These were randomly distributed in 5 focus groups. The sessions were recorded, transcribed and later analyzed. This allowed the identification of categories, subcategories and thematic units. The results show that the adolescents' main opinions and concerns on theatre tend to focus on the influence of theatre on their own personal development, self-confidence and emotional competence (individual level) and on the impact that dramatic activities have on their personal and empathic skills (social level). Finally, the results are discussed in terms of education, in relation to both curricular design and teaching methodology.

Keywords: theatre; adolescence; positive development; focus groups; qualitative methodology.

\section{EL TEATRE COM A RECURS PER AFRONTAR ELS REPTES DE L'ADOLESCÈNCIA}

Resum: Aquest estudi, utilitzant una metodologia d'investigació qualitativa, intenta aprofundir en les creences implícites $i$ percepcions que tenen els adolescents $i$ els joves sobre les seves experiències com a participants actius en activitats teatrals. Aquest article s'emmarca en un projecte d'investigació (El Teatre i els Joves) estructurat en diverses fases i que combina metodologies quantitatives i qualitatives. Un total de 53 participants dedats compreses entre els 12 i els 20 anys van participar en aquest estudi. Es van distribuir a latzar en 5 grups de discussió. Les sessions de treball es van gravar, transcriure $i$ analitzar posteriorment, cosa que va permetre la identificació de categories, subcategories $i$ unitats temàtiques. Els resultats posen de manifest que les principals opinions i preocupacions dels adolescents vers el teatre giren al voltant de la influència sobre el seu desenvolupament personal, la seva autoconfiança $i$ competència emocional (pla individual) $i$ sobre lefecte que tenen les activitats dramàtiques sobre les seves habilitats personals $i$ empàtiques (pla social). Finalment, sanalitzen els resultats en clau educativa, tant en làmbit de disseny curricular com de metodologia docent.

Paraules clau: teatre; adolescència; desenvolupament positiu; grups de discussió; metodologia qualitativa. 


\section{Introducción}

Desde la óptica de la educación, el teatro ha de ser considerado en su dimensión tanto artística como pedagógica. La experiencia teatral pone a sus participantes en situación de desplegar y practicar su potencialidad creativa, utilizando la integración de los distintos lenguajes (corporal, verbal, plástico, rítmico musical) desde un enfoque interdisciplinar o transdisciplinar (Motos, 2009). Ver y hacer teatro (Deldime, 1995) son dos perspectivas complementarias. El presente trabajo se focaliza en el hacer y pretende indagar en la opinión y el impacto que ocasiona en los adolescentes el hecho de tomar parte en actividades teatrales y los beneficios que, por ello, los propios protagonistas perciben sobre su desarrollo personal.

La adolescencia es un momento muy especial de transición entre la infancia y la edad adulta, en el que se construyen identidades y se formaliza la incorporación a la vida social más amplia. Cada nueva generación de adolescentes:

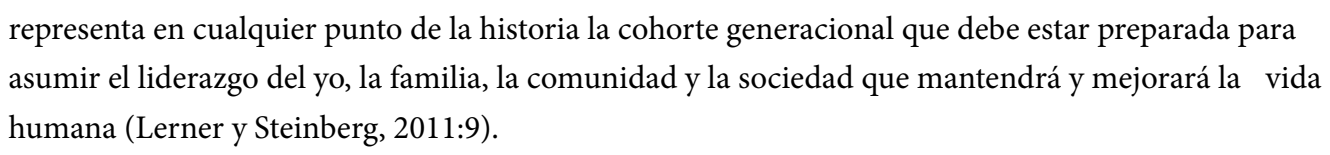

Los científicos tienen un papel vital para aumentar, a través de la generación de conocimiento básico y aplicado, la probabilidad de que los adolescentes se conviertan en ciudadanos plenamente comprometidos, implicados y capaces en la realización de estas contribuciones.

Desde el enfoque de la psicología positiva (Seligman, 2002), se entiende el desarrollo personal como la realización de uno mismo. Hughes y Wilson (2004) sostienen que este hace referencia a las habilidades, capacidades y recursos que ayudan a los jóvenes a hacer una transición exitosa a la adultez, es decir, llevar una vida saludable, confiada e independiente en la que poner de manifiesto su potencial. La literatura sobre el desarrollo positivo juvenil sugiere que el estudio de las interacciones sociales y de las actividades de ocio con los pares, amigos y otros grupos puede ayudar a entender mejor cómo se refuerzan los factores naturales de protección en la juventud de nuestros días. Hay muchas pruebas que demuestran que existe una estrecha relación entre el desarrollo de la juventud y las actividades extracurriculares en el ámbito del asesoramiento psicológico (Tonkins et al., 2005) y del teatro en la educación (Belliveau, 2005; Lazarus, 2012; Navarro, 2013).

La psicología positiva insiste en la construcción de competencias. Autores como O’Neill (1995), Wagner (2002), Baldwin, Fleming y Neelands (2003), Laferrière y Motos (2003), y Navarro (2013) dan cuenta de las posibilidades de las formas dramáticas como instrumento eficaz para desarrollar aspectos ligados a las competencia lingüística, cultural y artística, social y ciudadana, para aprender a aprender y desarrollar autonomía e iniciativa personal. Y en esta última destacan aspectos tales como superación de la timidez, conocimiento y aceptación personal, refuerzo de la autoestima, desarrollo de la creatividad y la expresión y la comunicación corporal y verbal. Y en cuanto a la competencia social y ciudadana, enfatizan los aspectos de conocimiento de otras personas y la mejora de las relaciones interpersonales, el trabajo en grupos y la organización y planificación del trabajo colectivo. 
Recientes enfoques del drama en la educación se centran en estudiar el impacto del teatro y las actividades dramáticas en el aprendizaje social y moral y en fomentar el sentido de pertenencia, confianza y seguridad y resiliencia. Así, Hanrahan y Banerjee (2017) y Elliott y Dingwall (2017) investigaron el impacto socioemocional del teatro en jóvenes en situación de riesgo. Gallagher y Rodricks, (2017) se centran en cómo ayuda el teatro aplicado a desarrollar una ética relacional en la educación y a tomar conciencia sobre las cuestiones de género. Phillips (2016) plantea que a través del drama se puede luchar contra la heterofobia para subvertir la hegemonía heterosexual. Por su parte, Needlands $(2008,2009)$ defiende que la importancia real del teatro con jóvenes y adolescentes reside en los procesos de implicación y participación social y artística y en la experimentación de una serie de situaciones y vivencias, más que en los resultados o productos artísticos que puedan ser elaborados.

Son escasos los estudios destinados a analizar el impacto de la actividad teatral en el desarrollo personal y social de los jóvenes. Hughes y Wilson (2004) trabajaron con trescientos jóvenes de edades comprendidas entre los 12 y los 30 años y encontraron que estos afirman que participar en actividades teatrales les ayuda a desarrollar su sentido de identidad, iniciativa, confianza, a tomar riesgos y a acrecentar la habilidad para expresarse. Según estos autores, el teatro ofrece a los jóvenes caminos que implican retos que demandan creatividad y suscitan incertidumbre y riesgo, pero en un entorno que ellos perciben como benigno y seguro y en el que pueden probar diferentes formas de ser y responder a los demás.

Rodríguez y Araya (2009) investigaron los efectos de la improvisación teatral y la expresión corporal sobre el desarrollo de los jóvenes y concluyeron que esta práctica tiene efectos positivos y significativos sobre el estado de ánimo de los participantes así como en su autoestima y confianza.

En definitiva, la influencia del teatro en el desarrollo personal, fundamentalmente durante la adolescencia y la adultez temprana, es un área que precisa de nuevos estudios e instrumentos que sirvan para describir, clarificar y explicar los potenciales efectos beneficiosos que la actividad teatral ejerce sobre el desarrollo en la juventud. Es necesario recopilar y analizar más evidencias sobre el impacto del teatro en el desarrollo personal que sirvan para construir una sólida base teórica que explique el cómo y el porqué de dicho efecto en los jóvenes (Hughes y Wilson, 2004).

Con el ánimo de contribuir a llenar ese vacío, Motos y Alfonso-Benlliure (2017) diseñaron y validaron la Batería El Teatro y Yo (TyY), instrumento que evalúa las creencias implícitas y percepciones que los jóvenes tienen sobre su participación en actividades teatrales y el impacto percibido sobre sus vidas. Utilizando el citado instrumento, Motos, Alfonso-Benlliure y Fields (2018) llevaron a cabo un estudio cuantitativo con 305 jóvenes vinculados con la práctica teatral para comparar las creencias de estos según edad y género. El estudio constató que los más mayores consideran que el impacto de la experiencia teatral es más intenso, que les ayuda a asumir riesgos y a tener una mayor apertura mental. Con respecto al género, las chicas obtuvieron puntuaciones más elevadas en dimensiones como "Tolerancia al riesgo y compromiso", "Identidad y autoconsciencia" y "Expresión de sentimientos" y también percibieron un mayor impacto de la experiencia teatral en sus vidas que los chicos.

El estudio que aquí se presenta, de carácter cualitativo, se centra en la recopilación y aná- 
lisis de las percepciones de los adolescentes implicados en actividades dramáticas a través de su participación en grupos de discusión y el análisis sistemático de sus valoraciones subjetivas sobre la vivencia teatral y la huella percibida sobre sus trayectorias evolutivas.

\section{Metodología}

\subsection{Participantes}

Un total de 53 sujetos de edades comprendidas entre los 12 y los 20 años (promedio: 15,6 y desviación estándar: 1,69) participaron en el estudio. El 29\% eran chicos y el $71 \%$, chicas. Procedían de 15 institutos de la ciudad de Valencia y de pueblos del área de influencia, 12 públicos y 3 privados concertados. Todos ellos estaban vinculados con el estudio y la práctica del teatro, ya fuera por cursar el Bachillerato Artístico, la asignatura optativa de Taller de Dramatización en la Educación Secundaria Obligatoria, o bien, por participar en algún taller extracurricular de teatro.

Los sujetos tomaron parte de los grupos de discusión durante el II y III Encuentro de Teatro en la Educación, celebrado en la Sala Inestable de Valencia. En la investigación se han tenido en cuenta los principios de la ética, pues a los participantes se les informaba y se les pedía el consentimiento para utilizar las grabaciones en una investigación posterior, manteniendo el anonimato.

\subsection{Procedimiento}

Los participantes fueron distribuidos al azar en 5 grupos de discusión, técnica de recogida de datos de naturaleza cualitativa que ha sido utilizada ampliamente en diferentes campos de la investigación sociológica.

Las sesiones de trabajo fueron grabadas y tras su transcripción, los datos, sometidos a análisis utilizando el programa informático Atlas.ti 7. En el proceso de codificación no existe ninguna fórmula para identificar las unidades temáticas y agruparlas en subcategorías y categorías: "hay que aprender a buscar temas examinado los datos de todos los modos posibles" (Taylor y Bogdan, 1987:160). La familia o categoría, agrupaciones de segundo grado de abstracción, no es más que la etiqueta que engloba a todos aquellos elementos aparecidos en el discurso que tienen rasgos comunes de significado. Por su parte, las subcategorías, agrupaciones de primer grado, concretan y limitan el significado de las categorías. Las unidades temáticas son fragmentos de texto, frases o incluso palabras que transmiten un contenido semántico relacionado con las preguntas estímulo formuladas por el moderador. Estas versaron sobre: razones por las que se participa en actividades teatrales; beneficios personales y mejoras obtenidas; si ayuda a expresar y gestionar mejor los sentimientos; compromiso; si favorece conocerse mejor y a los demás; ambiente en el grupo o en las clases de teatro; si coadyuva a evadirse de los problemas; si mejora las relaciones con los compañeros; y si lo aprendido en el teatro se transfiere a otros ámbitos. 


\section{Resultados}

El análisis de la información recogida en los grupos de discusión permitió la elaboración de determinadas categorías, subcategorías y unidades temáticas. En la tabla 1 se recogen los resultados del proceso de codificación.

\begin{tabular}{|c|c|c|c|}
\hline CATEGORÍAS & SUBCATEGORÍAS & UNIDADESTEMÁTICAS & PORCENTAJE \\
\hline \multirow[t]{5}{*}{ Beneficios } & CPB & 209 & $19,56 \%$ \\
\hline & EV & 41 & $3,84 \%$ \\
\hline & TRV & 62 & $5,80 \%$ \\
\hline & Total & 312 & $29,21 \%$ \\
\hline & PRE & 52 & $4,86 \%$ \\
\hline \multirow[t]{4}{*}{ Identificación } & ED & 52 & $4,86 \%$ \\
\hline & INS & 42 & $3,93 \%$ \\
\hline & THT & 35 & $3,27 \%$ \\
\hline & Total & 181 & $16,92 \%$ \\
\hline \multirow[t]{3}{*}{ Inicios } & ALT & 23 & $2,15 \%$ \\
\hline & INT & 29 & $2,71 \%$ \\
\hline & Total & 52 & $4,76 \%$ \\
\hline \multirow[t]{3}{*}{ Pensamiento } & $\mathrm{RHT}$ & 62 & $5,80 \%$ \\
\hline & PT & 91 & $8,52 \%$ \\
\hline & Total & 153 & $14,33 \%$ \\
\hline \multirow[t]{3}{*}{ Desafíos } & RTR & 70 & $6,55 \%$ \\
\hline & COM & 53 & $4,96 \%$ \\
\hline & Total & 123 & $11,52 \%$ \\
\hline \multirow[t]{3}{*}{$\begin{array}{c}\text { Autoconciencia } \\
\text { emocional }\end{array}$} & SEG & 40 & $3,75 \%$ \\
\hline & EXS & 67 & $6,27 \%$ \\
\hline & Total & 107 & $10,02 \%$ \\
\hline \multirow[t]{4}{*}{$\begin{array}{l}\text { Relaciones } \\
\text { personales }\end{array}$} & RAM & 60 & $5,62 \%$ \\
\hline & APY & 80 & $7,49 \%$ \\
\hline & Total & 140 & $13,11 \%$ \\
\hline & Total & 1068 & $100,00 \%$ \\
\hline
\end{tabular}

Nota: CPB: Cambio Personal; EV: Evasión; TRV: Transferencia; PRE: Presentación; ED: Edad; INS: Instituto; THT: Tiempo haciendo teatro; ALT: Aprendizaje del Lenguaje Teatral; INT: Iniciación al teatro; RHT: Razones para hacer teatro; PT: Pensamientos sobre el teatro; RTR: Retos y Riesgos; COM: Compromiso; SEG: Seguridad; EXS: Expresión de sentimientos; RAM: Relaciones de amistad; APY: Apoyo.

Tabla 1. Categorización y porcentajes. Elaboración propia. 


\subsection{Beneficios}

Lo más notorio del análisis de los datos es que los participantes en los grupos de discusión resaltan la utilidad que tiene para su desarrollo personal el hecho de hacer teatro o participar en actividades dramáticas. Y así, esta categoría, que alcanza casi el 30\% de las unidades temáticas, se refiere a las mejoras notadas y atribuidas a estas prácticas; a la transferencia que, de lo aprendido en teatro, hacen a su vida escolar, familiar y social; y a la percepción de tales actividades como un espacio de evasión de los problemas personales.

Dentro de esta primera categoría destaca la subcategoría "Cambio personal" (CPB) con aproximadamente una de cada cinco del total de las unidades temáticas. Es la más importante de todas ellas, no solo por el número total de unidades que comparten un significado común sino también por la centralidad que ocupa en el mapa de relaciones (ver Figura 1). Es la causa de las razones para hacer teatro y está asociada con el pensamiento sobre el teatro, la expresión de sentimientos, el sentimiento de seguridad, la evasión, el compromiso y la transferencia.

Entre los cambios personales que los participantes manifiestan haber experimentado destacan los siguientes: perder la vergüenza a actuar o hablar, lo que conduce a los participantes a declararse más abiertos y desinhibidos ("Yendo a teatro pierdes completamente la vergüenza"), sentirse más abiertos y comunicativos ("El teatro me ha ayudado a ser menos tímido") y tener mayor confianza en ellos mismos y en los demás. Y el incremento de esa confianza supone una mejora en la autoestima y en la capacidad para expresarse.

Los participantes manifiestan que el teatro es una oportunidad para conocerse mejor y les ayuda incluso, "a ser mejor persona". Es decir, es un medio para la construcción de la identidad, pues ayuda a los adolescentes "a ser más yo".

Y ello conduce a un aumento de la idea de satisfacción y a sentirse mejor consigo mismo. El teatro también les permite tomar conciencia de que otras personas tienen enfoques distintos y contribuye a poder ponerse en el punto de vista del otro ("Te ayuda a que pienses en los demás y veas cómo se sienten").

La segunda subcategoría, con un 5,80\%, es “Transferencia” (TRV). Esta se refiere a que tomar parte en actividades teatrales de alguna forma proporciona habilidades y recursos que luego pueden ser empleados en otros espacios y momentos de la vida de los practicantes. En este sentido, manifiestan que es "un aprendizaje continuo" y que "se lleva de la escena a la calle". Los participantes transfieren fundamentalmente lo aprendido en la clase de teatro o en el taller a las situaciones de aula o las interacciones sociales en el centro de enseñanza. 


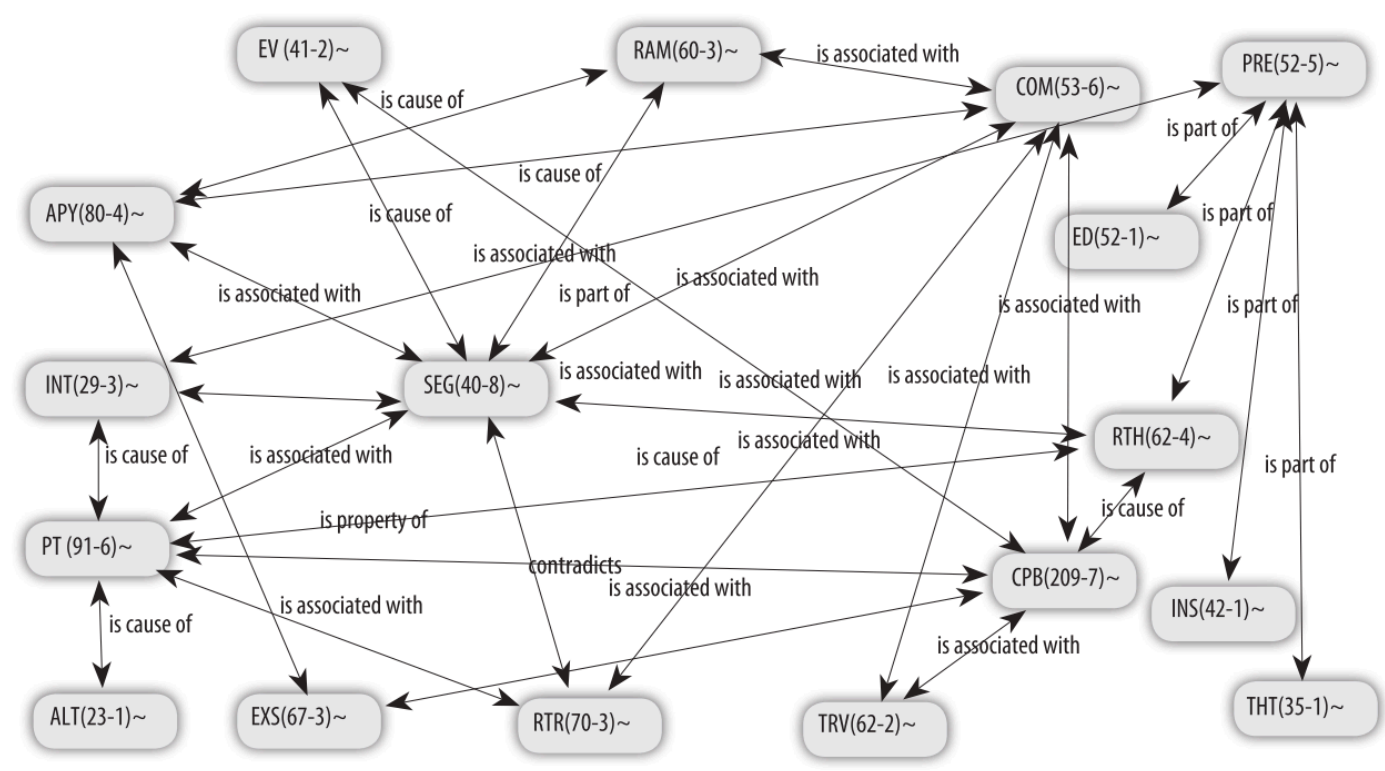

Figura 1. Red de relaciones de subcategorías. Elaboración propia.

La tercera subcategoría, con un 3,84\%, es "Evasión" (EV). El teatro es un espacio para olvidar y escapar por unas horas de los problemas personales. $\mathrm{Y}$, en este sentido, de alguna forma libera ("Dejo de pensar en cosas que me han pasado durante el día, en el instituto u otras cosas"), ('Yo tengo ganas de empezar teatro, porque es entrar y decir 'adiós problemas', porque es un sitio donde disfrutas, sonríes, te lo pasas bien. Es como que los problemas se van disolviendo, se van diluyendo"). Los participantes en el taller de teatro como actividad extraescolar lo conciben como una oportunidad para relajarse de la presión de las tareas que los estudios conllevan.

\subsection{Identificación}

La segunda categoría en cuanto a números de unidades temáticas (16,92\%) se refiere a datos de individualización de los participantes y reúne cuatro subcategorías: presentación, edad, instituto y tiempo transcurrido desde que el participante comenzó a formar parte de un grupo de teatro o se inició en las actividades dramáticas.

La subcategoría “Tiempo haciendo teatro" (THT) constituye el 3,27\% del total de las unidades temáticas. Algunos precisan con exactitud el tiempo que llevan con el teatro, que va desde los 9 meses (10 sujetos) a los 9 años ( 3 sujetos). Otros se limitan a expresarlo de una forma más imprecisa.

\subsection{Pensamiento sobre el teatro}

La tercera categoría, que supone un 14,33\% del total, agrupa las unidades de significado referidas a la idea que los participantes tienen sobre qué es teatro y las razones por las que han elegido las 
actividades teatrales, ya sea como asignatura optativa o como taller extracurricular.

La subcategoría "Pensamiento sobre el teatro" (PT) representa el 5,80\% de las unidades temáticas y reúne no solo lo que los participantes piensan qué es el teatro sino también qué rasgos ha de tener el teatro para jóvenes.

La idea que tienen los participantes sobre el teatro es algo muy amplio y difuso, como "meterte en la vida de otras personas y tener como otra vida", "olvidar quien eres y representar a otros", "un hobby", "un trabajo".

Los participantes asocian el concepto teatro con "expresión", "vida", "emoción", "ilusión", "libertad", "diversión", "cultura", etc. Algunos lo relacionan con "algo muy gratificante" y con la subida de adrenalina que se siente durante o después de las representaciones ("Son sentimientos que solo he experimentado haciendo funciones de teatro y es indescriptible").

También entienden el teatro como instrumento para educar en la aceptación de las diferencias y como un instrumento de cambio ("el teatro, sobre todo el social, ayuda a que poco a poco se vaya superando el miedo a lo diferente").

El teatro para los jóvenes ha de tener algunos de estos rasgos: "un lenguaje que te atraiga", "que esté planteado de una forma que nos interese", "que haya cambio de situaciones", etc. Y en cuanto a los temas: "la adolescencia", "con sus peleas entre amigas, el amor...", "los estudios" o "conflictos de la vida cotidiana".

"Razones para hacer teatro" (RHT). Esta subcategoría representa el 5,8\% de las unidades temáticas. La respuesta más frecuente cuando se plantea la cuestión por la que hacen teatro es porque "me gusta", "es algo necesario", "me siento bien" y "me siento a gusto". Otra que destacan es que el teatro es "una actividad diferente" y "divertida". Y añaden otras razones como "superar un poco la vergüenza". También declaran que es una oportunidad y un medio para conocerse mejor a sí mismo y para superar ciertas carencias.

Es conveniente distinguir entre quienes cursan la asignatura optativa de Taller de Dramatización/Teatro y quienes forman parte de un grupo de teatro como actividad extraescolar (donde también estarían comprendidos los que cursan Bachillerato Artístico). Para los primeros, las razones suelen ser del tipo: "era la optativa que tocaba", "no hay deberes ni exámenes", "las demás son más difíciles, y esta no la suspendes", etc.

Los del segundo grupo declaran razones como: "de mayor, quiero ser actor", "era una buena ocasión para expresar mis sentimientos", "es una actividad que necesito hacer para sentirme completo".

Como hemos señalado arriba, esta subcategoría está relacionada con 'Iniciación al teatro' (INT); por ello, entre las razones de participar en actividades teatrales encontramos la importancia del grupo de iguales.

\subsection{Relaciones personales}

Esta categoría representa el 13,11\% del total de unidades temáticas. Reúne las referidas al sentimiento de apoyo percibido en el grupo y a las relaciones de amistad que en él se establecen. 
La subcategoría "Apoyo" (APY), que supone 7,49\% de la totalidad de las unidades temáticas, se refiere a la autoconciencia de sentirse apoyado por los compañeros, el director o directora del grupo y por el profesorado del centro.

Respecto al apoyo y aceptación por parte de los compañeros, muestran opiniones como: "Si a un compañero le falta algo o se va a quedar colgado siempre estamos ahí para echar una mano". También manifiestan la importancia del apoyo del profesorado. El sentirse respaldado favorece que la representación salga mejor. En cuanto al resto del alumnado de clase o del instituto, no perciben ayuda sino más bien actitudes de incomprensión.

"Relaciones de amistad" (RAM). Esta subcategoría representa el 5,62\% de las unidades temáticas. Se refiere a que el teatro es considerado como un medio que permite establecer vínculos distintos a las establecidos con las personas con que uno se relaciona en el día a día: "Completamente diferente hasta en el trato con los compañeros en el instituto". Con relativa frecuencia suelen utilizar metáforas como "piña" y "familia" para describir estas conexiones. El taller de teatro es un ámbito excelente para crear grupo y relaciones de cooperación. Este rasgo de sociabilidad específica es inherente al concepto grupo o compañía de teatro.

\subsection{Desafíos}

Esta categoría reúne el 11,52\% de las unidades temáticas y hace referencia a los desafíos que les presenta el participar en actividades teatrales y el compromiso que esto conlleva.

"Retos y riesgos" (RTR). Subcategoría que representa el 6,55\% del total de unidades temáticas. Se refiere a que hacer teatro y participar en las actividades de dramatización supone correr riesgos y responder a desafíos.

Uno de los retos principales del que tienen conciencia es hacer llegar claramente al público el mensaje que la obra o la dramatización contienen, atraer su atención y mantenerla y la preocupación por que se escuche claramente la voz de cada actor o actriz: "Cuando hacemos teatro, se nos plantea el reto de transmitir al público lo que queremos".

A los participantes les abate verse expuestos a los comentarios e, incluso, burlas de los compañeros del instituto. También concretan como riesgos la falta de confianza en sí mismos y los temores a "equivocarse" o "quedarse en blanco".

"Compromiso" (Com). La segunda subcategoría, con un 4,96\%, se refiere a que el teatro requiere tomar parte de un proceso de trabajo disciplinado y comprometido. Los participantes entienden el compromiso como que "todos tenemos que estar pendientes de todos" porque "actuar es compromiso". También lo ven como exigencia personal y tienen conciencia de que el compromiso implica esfuerzo continuado. Esta responsabilidad es "contigo mismo sobre todo y con el grupo" y con "con la obra en sî", con "la directora" y "con el público" porque "si lo haces mal, el público tampoco lo va a pasar bien". 


\subsection{Autoconciencia emocional}

Esta categoría, con el $10 \%$ de total de las unidades temáticas, se refiere a la oportunidad que el teatro ofrece para expresar sentimientos y pensamientos en un contexto seguro.

La subcategoría "Expresión de sentimientos" (EXS) supone el 6,27\% y reúne las unida-des temáticas que se refieren a la coyuntura que proporciona hacer teatro y actividades dramáticas para expresar sentimientos y pensamientos, pues es "donde puedes expresarte como eres".

Pero asimismo el teatro y las actividades teatrales permiten tomar conciencia de los senti-mientos, puesto que ayuda a no tener miedo a mostrar a los demás como uno se percibe ("sentirte bien contigo mismo"), lo que supone un crecimiento asertivo.

E igualmente ayuda a tomar conciencia de lo sentimientos de los demás. Manifiestan que el teatro les ha cambiado "la manera de pensar", "la manera de ser". Y de este modo lo conciben como un medio para establecer una identidad positiva.

Seguridad (SEG) Esta subcategoría, referida a la percepción de que el grupo de teatro supone un contexto seguro, solo supone el 3,75\% de las unidades temáticas. A pesar de su bajo porcentaje representa la centralidad, con ocho conexiones, en el mapa de las relaciones (ver figura 1). En este sentido, la expresión de sentimientos es una propiedad de la seguridad. Además, está asociada con la opinión que se tiene sobre el teatro, con la conciencia de apoyo, con el compro-miso, con las relaciones de amistad y con los cambios personales percibidos.

\subsection{Inicios}

La última categoría, pues solo recoge el 4,76\% de las unidades temáticas, se refiere tanto al ini-cio de la experiencia teatral práctica (teatro para hacer), al conocimiento del lenguaje, como a la recepción (teatro para ver) ya sea en el contexto familiar, escolar o en el marco de la formación académica.

\section{Discusión}

El presente trabajo se enmarca en un proyecto de investigación estructurado en varias fases y que combina metodologías tanto cuantitativas y cualitativas. En la segunda, en la que nos encontra-mos, el objetivo es identificar las creencias y percepciones de los adolescentes sobre el teatro y el impacto en sus vidas. En fases posteriores contrastaremos esas percepciones subjetivas con el desarrollo real y también lo compararemos con aquellos adolescentes que no entran en contacto con la experiencia teatral.

En líneas generales, en este estudio descriptivo sobre las creencias implícitas de los jóve-nes podemos extraer como primera conclusión que la idea que aparece con mayor consistencia en sus declaraciones es la de un efecto beneficioso de la experiencia teatral sobre sus vidas. Funda-mentalmente a dos niveles: primero, promueve un cambio personal; segundo, el teatro les ofrece la oportunidad de aprender unas habilidades que resultan importantes en otras esferas de sus vidas (transferencia de aprendizajęs). 
Por lo que respecta al cambio personal, los sujetos adolescentes manifiestan que las actividades dramáticas son una oportunidad para conocerse mejor y que les ayuda a "crecer como personas". Es decir, conceptualizan el teatro como una herramienta para la construcción de la identidad, entendida como un medio para alcanzar cierto sentido de autonomía e independencia.

Los jóvenes prueban diferentes identidades, a menudo bajo la influencia de sus iguales. Y como señala Kim (2015:72). "Las actividades teatrales se caracterizan por la libertad de expresión, por un espacio no institucionalizado y por la oportunidad de explorar o reinventar la propia identidad". Posiblemente, el mediador cognitivo que permite y facilita la exploración de la identidad a través de la práctica teatral es la apertura mental. Esta promueve y facilita el perspectivismo, la redefinición de los problemas, la toma de actitudes más abiertas y tolerantes con la información, las situaciones y las personas. Hacer teatro ayuda a los jóvenes a adoptar muchos roles, algo especialmente importante para el proceso y el desempeño creativo (Hughes y Wilson, 2004).

Por lo que se refiere a la transferencia de aprendizajes, los jóvenes valoran la experiencia teatral como fuente de aprendizajes que les van a ser útiles más allá de los espacios de la performance. Así pues, la importancia de la experiencia teatral trasciende la interpretación y se proyecta sobre futuros sentimientos de confianza y competencia.

Tras los beneficios percibidos, la siguiente categoría más relevante identificada en el análisis es el interés por la reflexión teórica y pragmática sobre el teatro como herramienta para el cambio. A pesar de que las ideas expuestas por los jóvenes pueden ser a veces radicales y otras veces ambiguas y difusas, denotan una gran preocupación y deseo de reflexión sobre el teatro y sus posibilidades. Es probable que ese entusiasmo y pasión que despierta el teatro en los jóvenes que lo practican, resida en una de sus características centrales: brindar una zona de "exploración sin sanción" (Heathcote, 1991). Lo que ocurre en el escenario, parece real, pero no lo es. En dicha zona los jóvenes pueden probar experiencias, pero sin tener que vivir sus consecuencias reales. En este sentido constata Davis (2015:72) "mientras que el contexto y el rol son ficticios, las emociones que se sienten pueden ser 'reales' a la vez que se alejan de 'lo real". Esto facilita que se puedan adoptar diferentes perspectivas y actitudes y comprometerse emocionalmente, debido a ello el teatro resulta muy atractivo para los adolescentes ávidos por explorar y conocer. Tal estado, consistente en pertenecer simultáneamente a dos mundos diferentes y autónomos (el real y el del drama), es llamado "metaxis" por Boal (2004:66).

El siguiente tema de interés para los jóvenes participantes son las relaciones con sus iguales que el teatro les brinda. En la adolescencia se inicia un proceso de individuación y autonomía de los progenitores y, en consecuencia, los amigos pasan a ser valorados como la principal fuente de influencia (Martínez, 2013). Las relaciones interpersonales y la sensación de apoyo y seguridad percibida respecto al grupo de referencia adquieren una importancia crucial y formar parte de grupos de teatro parece ayudar a la facilitación y el fortalecimiento de ese sentimiento de pertenencia y apoyo. Cuando el joven es capaz de establecer relaciones próximas con amigos y se siente satisfecho con estas relaciones, dispone de más recursos y seguridad para hacer frente a situaciones problemáticas. Formar parte de un grupo de teatro les proporciona una sensación de apoyo a varios niveles: informativo (consejo y orientación en la solución de problemas), instrumental 
(ayuda en tareas escolares, domésticas, materiales), de compañerismo (confianza en el otro y compartir las actividades sociales) y de estima (ánimo, consuelo, etc.). Wernick, Kulick y Woodford (2014) trabajando con un grupo de jóvenes gays, bisexuales y transexuales llegaron a la conclusión de que en estos colectivos, la implicación en actividades dramáticas favorecía la percepción del ambiente social como "seguro" y la sensación de apoyo y respeto como miembros del grupo.

Finalmente, el último apartado digno de mención es la autoconsciencia emocional. Los adolescentes "actores" entienden que el teatro también les permite probar sus propias reacciones emocionales, descubrir la diversidad de la "paleta de colores de las emociones" (autoconsciencia) y las múltiples posibilidades de canalización de dichas emociones (expresión emocional). De alguna manera, intuyen que el teatro promueve su inteligencia emocional al ayudarles a construir un espacio cognitivo entre la vivencia y la expresión emocional. La práctica teatral les ayuda a identificar emociones y reflexionar sobre ellas, y además, les ofrece una vía para canalizarlas saludablemente. Estas habilidades emocionales tienen una importancia determinante a la hora de predecir el ajuste psicosocial. Los adolescentes con mayor destreza emocional tienen mejores relaciones sociales, mayor percepción de sentirse queridos por los demás (padres e iguales), mayor confianza en sí mismos y mayor competencia percibida (Salguero, Fernández-Berrocal, Ruiz-Aranda, et al., 2011).

\section{Limitaciones y consideración final}

Por supuesto, la interpretación de los resultados del presente trabajo debe hacerse teniendo en cuenta sus limitaciones. Los grupos de discusión nos ofrecen una serie de ventajas (accesibilidad, vivencialidad, frescura) pero también ciertas restricciones (subjetividad, tendencia, pasión). En ocasiones, las declaraciones hechas por los sujetos fueron muy contundentes y carentes de matices, lo que puede considerarse un rasgo propio de la edad de los participantes.

Otra limitación es que los resultados están basados en datos aportados por participantes comprometidos en grupos de teatro. ¿Qué ocurriría si incluyéramos jóvenes que practicaron teatro y encontraron que el teatro no era para ellos?

En definitiva, parece ser que formar parte de un grupo implicado en procesos de creación teatral puede tener una influencia relevante en el desarrollo de la competencia intrapersonal e interpersonal. Los jóvenes así lo manifiestan y parecen convencidos de ello. En un momento vital repleto de dudas, tensiones e interrogantes por resolver en cuanto a la identidad personal y los roles sociales, el teatro puede ser un recurso y un espacio útil para tales tareas del desarrollo. Por supuesto, bajo la guía de los profesionales de la educación. Estos resultados permiten reivindicar la necesidad de ofrecer a los jóvenes materias curriculares fundamentadas en las artes escénicas que faciliten experiencias de autoconocimiento, desarrollo de los talentos, expresión emocional y construcción de una identidad positiva y saludable. Después de todo, como decía Vittorio Gassman, el teatro no se hace para contar cosas, sino para cambiarlas. 


\section{Referencias bibliográficas}

Baldwin, P., Fleming, K., y Neelands, J. (2003). Teaching literacy through drama: Creative approaches. London: Routledge Falmer.

Beare, D., y Belliveau, G. (2007). Theatre for positive youth development: A development model for collaborative play-creating. Applied Theatre Research, 8, 1-16. Recuperado de: https:// www.academia.edu/6301680/Theatre_for_Positive_Youth_Development_A_Development_Model_for_Collaborative_Play-creating

Belliveau, G. (2005). An arts-based approach to teach social justice: Drama as a way to address bullying in schools. International Journal of Arts Education, 3. Recuperado de: http://citeseerx.ist.psu.edu/viewdoc/download doi: 10.1.1.463.6105\&rep=rep1\&type=pdf

Boal, A. (2004). El arco iris del deseo. Barcelona: Alba.

Davis, S. (2015). Perezhivanie and experience of drama, metaxis and meaning making. Drama Australia Journal, 39(1), 63-75. doi: 10.1080/14452294.2015.1083138

Deldime, R. (1995) Théâtre: art vivant. Carnières-Morlanwelz: Lasman.

Elliott, V., y Dingwall, N. (2017) Roles as a route to being 'other': drama-based interventions with at-risk students. Emotional and Behavioural Difficulties, 22(1) 66-78. doi: http://doi.org/10.1080/13632752.2017.1290875

Gallagher, K., y Rodricks, D.J. (2017) Hope despite hopelessness: Race, gender, and the pedagogies of drama/applied theatre as a relational ethic in neoliberal times. Youth Theatre Journal, 31(2), 114-128. doi: 10.1080/08929092.2017.1370625

Hanrahan, F., y Banerjee, B. (2017) 'It makes me feel alive': The socio-motivational impact of drama and theatre on marginalised young people. Emotional and Behavioural Difficulties, 22(1), 35-49. doi: 10.1080/13632752.2017.1287337

Heathcote, D. (1991). Collected writings on education and drama. Evanston, IL: Northwestern University Press.

Hughes, J., y Wilson, K. (2004). Playing a part: the impact of youth theatre on young people's personal and social development. Research in Drama Education, 9(1), 57-72. doi: $10.1080 / 1356978042000185911$.

Kim, A., Stembridge, S., Lawrence, C., Torres, V., Miodrag, N., Lee, J., y Boynes, D. (2015). Neurodiversity on the stage: The effects of inclusive theatre on youth with autism. International Journal of Education and Social Science, 2(9), 27-39. Recuperado de: www.ijessnet.com/ wp-content/uploads/2015/10/4.pdf

Laferrière, G., y Motos, T. (2003). Palabras para la acción. Ciudad Real: Naque.

Lazarus, J. (2012). Sings of change: New directions in Theatre education. Chicago, IL: Intellect.

Lerner, R.M., y Steinberg (2011). Handbook of adolescence psychology (2a ed.) Hoboken, NY: John Wiley and Sons.

Martínez, B. (2013). El mundo social del adolescente: amistades y pareja. En E. Estevez (Coord.), Los problemas en la adolescencia: respuestas y sugerencias para padres y educadores (pp.7196). Madrid: Síntesis. 
Motos, T., y Alfonso-Benlliure, V. (2017). Diseño y validación de la batería "El Teatro y Yo": Evaluación del impacto de la experiencia teatral en los jóvenes. Crearmundos, 15, 346-352. Recuperado de http://www.crearmundos.net/asociacion/as/revista_files/Revista\%20 Creamundos\%2015_2017.pdf

Motos, T., Alfonso-Benlliure, V., \& Fields, D. L. (2018). The impact of theatrical experiences on young adults. Research in Drama Education (RIDE): The Journal of Applied Theatre and Performance. doi: 10.1080/13569783.2018.1494562.

Motos, T. (2009). El teatro en la Educación Secundaria. Fundamentos y retos. Creatividad y Sociedad, 14. Recuperado de http://www.creatividadysociedad.com/articulos/14/3-el_teatro_en_la_educ\%20secundaria_tomas_motos.pdf

Navarro, A. (2013). Teatro para el cambio. Nuevos enfoques del teatro en la educación. Ciudad Real: Ñaque. Recuperado de: http://www.naque.es/virtuemart/179/15/ebook/teatro-para-el-cambio-en-la-educacion-nuevos-enfoques-detail

Needlands, J. (2008). Essentially youth theatre. NAYAD Starting The Debate Seminar, May $10^{\text {th }}$ 2008. Recuperado de: http://nayad.ie./files/jNeedlandsadress.pdf

Neelands, J. (2009). Acting together: ensemble as a democratic process in art and life. Research in Drama Education: The Journal of Applied Theatre and Performance, 14(2), 173- 189. doi: $10.1080 / 13569780902868713$

O'Neill, C. (1995). Drama worlds. A framework for process drama. Toronto (ON): Pearson Education Canada

Phillips, H. (2016). Heterophobia: subverting heterosexual hegemony through intermedial applied performance for young people. Research in Drama Education: The Journal of Applied Theatre and Performance, 21(3), 319-331. doi: 10.1080/13569783.2016.1194190

Rodríguez, V., y Araya, G.A. (2007). Efecto de ocho clases de expresión corporal en el estado de ánimo y autoconcepto general de jóvenes universitarios. Revista de Educación, 33(2), 139152. Recuperado de: http://www.redalyc.org/pdf/440/44012058009.pdf

Seligman, M.E.P. (2002). Authentic happiness: Using the new positive psychology to realize your potential for lasting fulfillment. New York: Free Press/Simon and Schuster.

Salguero, J. M., Fernández-Berrocal, P., Ruiz-Aranda, D., Castillo, R., y Palomera, R. (2011). Inteligencia emocional y ajuste psicosocial en la adolescencia: El papel de la percepción emocional. European Journal of Education and Psychology, 4(2), 143-152. doi: https:// doi.org/10.1989/ejep.v4i2.84

Taylor, S. J., y Bogdan, R. (1987). Introducción a los métodos cualitativos de investigación (2a ed.). Barcelona: Paidós.

Tonkins, R.S., Murphy, A., Lee, Z., Saewyc, E., y The McCreary Centre Society (2005). British Columbia youth health trends: A retrospective, 1992-2003. Vancouver, BC: The McCreary Centre Society. Recuperado de: http://www.mcs.bc.ca/pdf/AHS-Trends-2005-report.pdf

Wagner, B. J. (2002). Understanding drama-based education. En G. Brauer (Ed.), Body and language: Intercultural learning through drama (pp. 3-18). Westport, CT: Ablex Publishing. 
Wernick, L. J., Kulick, A., y Woodford, M. R. (2014). How theater within a transformative organizing framework cultivates individual and collective empowerment among LGBTQQ youth. Journal of Community Psychology, 42(7), 838-853. doi: https://doi.org/10.1989/ejep.v4i2.84 